\title{
The Large Deviation Principle and Some Models of an Interacting Boson Gas
}

\author{
M. van den Berg ${ }^{1}$, J. T. Lewis ${ }^{2}$, and J. V. Pulé ${ }^{3}$ \\ 1 Department of Mathematics, Heriot-Watt University, Riccarton, Edinburgh, EH14 4AS, U.K. \\ 2 Dublin Institute for Advanced Studies, 10 Burlington Road, Dublin 4, Republic of Ireland \\ ${ }^{3}$ Department of Mathematical Physics, University College, Dublin 4, Republic of Ireland
}

\begin{abstract}
This is a study of the equilibrium thermodynamics of the HuangYang-Luttinger model of a boson gas with a hard-sphere repulsion using large deviation methods; we contrast its properties with those of the mean field model. We prove the existence of the grand canonical pressure in the thermodynamic limit and derive two alternative expressions for the pressure as a function of the chemical potential. We prove the existence of condensate for values of the chemical potential above a critical value and verify a prediction of Thouless that there is a jump in the density of condensate at the critical value. We show also that, at fixed mean density, the density of condensate is an increasing function of the strength of the repulsive interaction. In an appendix, we give proofs of the large deviation results used in the body of the paper.
\end{abstract}

\section{Introduction}

Since London's proposal [1] that the super-fluid phase-transition in $\mathrm{He}^{4}$ is an example of Bose-Einstein condensation, it has been of interest to know how, in theory, interparticle forces affect the condensation of bosons. London himself conjectured [2] that, as a manifestation of quantum mechanical complementarity, the momentum-space condensation of bosons is enhanced by spatial repulsion between the particles; we know of no proof of this general proposition.

Huang et al. [3] introduced a model of a boson gas with a hard-sphere repulsion which displays enhanced condensation. The model may be described thus: Let $\Lambda_{1}, \Lambda_{2} \ldots$ be a sequence of regions in $\mathbb{R}^{d}$ and denote the volume of $\Lambda_{l}$ by $V_{l}$; we assume that $V_{l} \rightarrow \infty$ as $l \rightarrow \infty$. We associate with the region $\Lambda_{l}$ the sequence $\varepsilon_{l}(1) \leqq \varepsilon_{l}(2) \leqq \ldots$ of ordered real numbers, where $\varepsilon_{l}(j)$ is the $j^{\text {th }}$ eigenvalue of the single particle hamiltonian of the non-interacting system in the region $\Lambda_{l}$; the freegas hamiltonian $H_{l}^{0}$ is given by

$$
H_{l}^{0}=\sum_{j \geqq 1} \varepsilon_{l}(j) n_{l}(j),
$$


where $n_{l}(j)$ is the occupation number of the $j^{\text {th }}$ level. The Huang-Yang-Luttinger model is described by the hamiltonian

$$
H_{l}^{\mathrm{HYL}}=H_{l}^{0}+\frac{a}{2 V_{l}}\left\{2 N_{l}^{2}-\sum_{j \geqq 1} n_{l}(j)^{2}\right\},
$$

where $N_{l}=\sum_{j \geqq 1} n_{l}(j)$ is the total number of particles and $a>0$. The HYL-model is to be compared with the mean-field model described by the hamiltonian

$$
H_{l}^{\mathrm{MF}}=H_{l}^{0}+\frac{a}{2 V_{l}} N_{l}^{2} .
$$

In the mean-field model, the interaction may be understood classically: the term $\frac{a}{2 V_{l}} N_{l}^{2}$ is an "index of refraction" approximation to the interaction energy in which we imagine each particle to move through the system as though it were moving in a uniform optical medium and so receiving an increment in energy proportional to $\frac{N_{l}}{V_{l}}$. The HYL-hamiltonian differs from the mean-field hamiltonian by the term

$$
\frac{a}{2 V_{l}}\left\{N_{l}^{2}-\sum_{j \geqq 1} n_{l}(j)^{2}\right\}
$$

which is a purely quantum mechanical contribution to the interaction energy and reflects the boson statistics. It is smallest when all the particles are in the same energy-level; we expect, therefore, that any tendency to condense in momentum space which is displayed by the free-gas will be enhanced by the presence of this term in the interaction.

Huang et al. [3] motivated the introduction of their model by reference to firstorder perturbation theory. Thouless, in his book [4], offered an alternative approach: he started from the full pair-potential interaction, and, as a first step, discarded those terms which are not diagonal in the occupation numbers $\left\{n_{l}(j)\right.$ : $j=1,2, \ldots\}$; the remaining terms can be grouped as the mean-field part, the term (1.4) and a correction term involving the pair-potential function. Formally, the correction term vanishes in the delta-function limit so that we recover the HYLhamiltonian, while in the van der Waals' limit it cancels with the term (1.4) to give the mean-field hamiltonian; details of this can be found in [5].

While arguments which point to a resemblance between the HYL-model and an actual hard-sphere gas are only heuristic, the properties of the model are nevertheless of interest provided their derivation is rigorous; the method of large deviations, Varadhan's theorem [6] in particular, enable us to give such proofs. Huang et al. [3] argued that the condensate, if any, would occupy the ground state and concluded that (1.4) could be replaced by

$$
\frac{a}{2 V_{l}}\left\{N^{2}-n_{l}(1)^{2}\right\}
$$

They then used the method of the largest term (Laplace's method) to obtain an expression for the free-energy; their derivation gives the following expression for 
the grand canonical pressure $p^{\mathrm{HYL}}(\mu)$ :

$$
p^{\mathrm{HYL}}(\mu)=\sup _{0 \leqq x_{1} \leqq x_{0}}\left\{\mu x_{0}-f\left(x_{0}-x_{1}\right)-\frac{a}{2}\left(2 x_{0}^{2}-x_{1}^{2}\right)\right\},
$$

where $f(\varrho)$ is the canonical free-energy density of the free boson gas at density $\varrho$. In Sect. 4 , we give a rigorous derivation of (1.6) starting from the hamiltonian

$$
H_{l}^{\mathrm{HYL}}=H_{l}^{0}+\frac{a}{2 V_{l}}\left\{2 N^{2}-\sum_{j=1}^{m_{l}} n_{l}(j)^{2}\right\},
$$

where $\left\{m_{l}: l=1,2, \ldots\right\}$ is any sequence of positive integers satisfying

$$
\lim _{l \rightarrow \infty} m_{l} / V_{l}=0 .
$$

[Ideally, we would like to remove the condition (1.8); we have been unable to surmount the technical problems which this change introduces.] The only conditions we require on the free-gas hamiltonians $H_{l}^{0}$ are those introduced in [7] to ensure the existence of the free-gas pressure; for example, the cases in which the single-particle hamiltonian has a spectral gap above the ground state [8] or includes an external potential [9] are covered by this treatment. We give also (in Sect. 6) a full discussion of the phenomenon of condensation in the HYL-model, using the method introduced in [10]; this requires an explicit expression for the pressure in terms of the integrated density of states of the single-particle hamiltonian, and we provide this in Sect. 5 by solving the variational problem (1.6).

The first step in our proof is to bound the interaction term (1.6) above by

$$
\frac{a}{2 V_{l}}\left\{N_{l}^{2}-n_{l}(1)^{2}\right\}
$$

and below by

$$
\frac{a}{2 V_{l}}\left\{N_{l}^{2}-\left(\sum_{j=1}^{m_{l}} n_{l}(j)\right)^{2}\right\}
$$

The principle of large deviations provides a compact way of making rigorous the method of the largest term; applied to the upper bound on the hamiltonian it yields the expression (1.6) for a lower bound to the pressure $p^{\mathrm{HYL}}(\mu)$. To deal with the lower bound (1.9) we have first to estimate the entropy involved in grouping together the first $m_{l}$ levels; this is achieved by an inequality proved in Sect. 4; the method of large deviations applied to the lower bound (1.9) then yields the expression (1.6) for an upper bound to the pressure $p^{\mathrm{HYL}}(\mu)$ and the proof is complete.

The method of large deviations is still not well-known in the theoretical physics community; for this reason, we introduce the technique first in Sect. 3 by means of a simple example, using it to prove the existence of the pressure in the mean-field model.

For the convenience of the reader, we close this section with a summary of those results on the free-boson gas, proved in [7], which we will make use of in this 
paper. A minor, but technically important remark has to be made at this point: the thermodynamics of each of the three models described here depends only on the subtracted eigenvalues

$$
\lambda_{l}(j)=\varepsilon_{l}(j)-\varepsilon_{l}(1)
$$

and not on the eigenvalues $\varepsilon_{j}(j)$ themselves; this is discussed fully in [7] for the case of the free gas and the discussion carries over easily to the HYL and mean-field models. This being said, the free-gas pressure $p_{l}(\mu)$ in $A_{l}$ at chemical potential $\mu$ can be written, for $\mu<0$, as

$$
p_{l}(\mu)=\left(\beta V_{l}\right)^{-1} \sum_{j \geqq 1} \ln \left(1-e^{\beta\left(\mu-\lambda_{l}(j)\right)}\right)^{-1} .
$$

Defining the partial pressure

$$
p(\mu \mid \lambda)=\beta^{-1} \ln \left(1-e^{\beta(\mu-\lambda)}\right)^{-1}
$$

and the distribution function

$$
F_{l}(\lambda)=\left(V_{l}\right)^{-1} \#\left\{j: \lambda_{l}(j) \leqq \lambda\right\},
$$

we may re-write (1.11) as

$$
p_{l}(\mu)=\int_{[0, \infty)} p(\mu \mid \lambda) d F_{l}(\lambda) .
$$

To ensure the convergence of the sequence $\left\{p_{l}(\mu)\right\}$ we must impose some conditions on the single particle spectrum. Define

$$
\phi_{l}(\beta)=\int_{[0, \infty)} e^{-\beta \lambda} d F_{l}(\lambda),
$$

and introduce the conditions

(S1): $\phi(\beta)=\lim _{l \rightarrow \infty} \phi_{l}(\beta)$ exists for all $\beta$ in $(0, \infty)$.

$(\mathrm{S} 2): \phi(\beta)$ is non-zero for at least one value of $\beta$ in $(0, \infty)$.

When (S1) holds, there exists a unique distribution function $F$ such that

$$
\phi(\beta)=\int_{[0, \infty)} e^{-\beta \lambda} d F(\lambda)
$$

and $F_{l}(\lambda) \rightarrow F(\lambda)$, at least at the points of continuity of $F$. The function $F$ is the integrated density of states. When in addition (S2) holds, we can say more:

Proposition 1. Suppose that (S1) and (S2) hold; then the limit

$$
p(\mu)=\lim _{l \rightarrow \infty} p_{l}(\mu)
$$

exists for $\mu>0$, and $p(\mu)$ is given by

$$
p(\mu)=\int_{[0, \infty)} p(\mu \mid \lambda) d F(\lambda) .
$$

The critical density $\varrho_{c}$ is defined as follows: if $\lambda \mapsto p^{\prime}(0 \mid \lambda)$ is integrable on $[0, \infty)$ with respect to $F$, put

$$
\varrho_{c}=\int_{[0, \infty)} p^{\prime}(0 \mid \lambda) d F(\lambda)
$$


put $\varrho_{c}=\infty$ otherwise. For fixed $l$, the function $\mu \mapsto p_{l}^{\prime}(\mu)$ is strictly increasing on $(-\infty, 0)$ and $p_{l}^{\prime}(\mu) \rightarrow 0$ as $\mu \rightarrow-\infty$ while $p_{l}^{\prime}(\mu) \rightarrow \infty$ as $\mu \rightarrow 0$ since $\lambda_{l}(1)=0$. It follows that the equation $p_{l}^{\prime}(\mu)=\varrho$ has a unique solution $\mu_{l}(\varrho)$ in $(-\infty, 0)$ for each $\varrho$ in $(0, \infty)$. On the other hand, the function $\mu \mapsto p^{\prime}(\mu)$ increases from zero to $\varrho_{c}$ as $\mu$ ranges through $(-\infty, 0)$. It is convenient to define $\mu(\varrho)$ for $\varrho$ in $(0, \infty)$ as the unique root of $p^{\prime}(\mu)=\varrho$ if $\varrho<\varrho_{c}$ and zero if $\varrho \geqq \varrho_{c}$. Defining $\pi_{l}(\varrho)$, the pressure at mean density $\varrho$, by

$$
\pi_{l}(\varrho)=\left(p_{l} \circ \mu_{l}\right)(\varrho)
$$

and $\pi(\varrho)$ by

$$
\pi(\varrho)=(p \circ \mu)(\varrho)
$$

we have:

Proposition 2. Suppose that (S1) and (S2) hold; then

$$
\begin{aligned}
& \lim _{l \rightarrow \infty} \mu_{l}(\varrho)=\mu(\varrho), \\
& \lim _{l \rightarrow \infty} \pi_{l}(\varrho)=\pi(\varrho) .
\end{aligned}
$$

The canonical free-energy $f(\varrho)$ at mean density $\varrho$, defined by $f(\varrho)$ $=\sup _{\mu<0}\{\mu \varrho-p(\mu)\}$, is given in terms of $\mu(\cdot)$ and $\pi(\cdot)$ by

$$
f(\varrho)=\varrho \mu(\varrho)-\pi(\varrho) .
$$

Notice that $\varrho \mapsto f(\varrho)$ is constant on the segment $\left[\varrho_{c}, \infty\right)$ so that, for $\varrho_{c}<\infty$, there is a first-order phase-transition.

In the proof of the large deviation result in the Appendix, we require a more technical result: Define

$$
\begin{aligned}
& p^{(0)}(\mu \mid \lambda)=p(\mu \mid \lambda), \\
& p^{(k)}(\mu \mid \lambda)=\frac{d^{k}}{d \lambda^{k}} p(\mu \mid \lambda), \quad k \geqq 1,
\end{aligned}
$$

we have

Proposition 3. Suppose that (S1) and (S2) hold; then for each $k=0,1,2, \ldots$ and each $\mu>s$ the sequence $p_{l}^{(k)}(\mu ; s) \stackrel{\text { def }}{=} \int_{[s, \infty)} p^{(k)}(\mu \mid \lambda) d F_{l}(\lambda)$ converges to

$$
p^{(k)}(\mu ; s) \stackrel{\text { def }}{=} \int_{[s . \infty)} p^{(k)}(\mu \mid \lambda) d F(\lambda),
$$

provided s is a point of continuity for $F$. Moreover, the convergence is uniform in $\mu$ on compacts in $(-\infty, s)$.

Defining

$$
f(\varrho ; s)=\sup _{\mu<s}\{\mu \varrho-p(\mu ; s)\}
$$

and

$$
\lambda_{0}=\inf \{\lambda: F(\lambda)>0\}
$$


we have

$$
f\left(\varrho ; \lambda_{0}\right)= \begin{cases}\varrho \mu(p)-p\left(\mu(\varrho) ; \lambda_{0}\right), & 0 \leqq \varrho \leqq \varrho_{n}, \\ \lambda_{0} \varrho-p\left(\lambda_{0} ; \lambda_{0}\right), & \varrho_{n} \geqq \varrho,\end{cases}
$$

where

$$
\varrho_{n}=\int_{\left[\lambda_{0}, \infty\right)} p^{\prime}\left(\lambda_{0} \mid \lambda\right) d F(\lambda) \geqq \int_{\left[\lambda_{0}, \infty\right)} p^{\prime}(0 \mid \lambda) d F(\lambda)=\varrho_{c}
$$

and $\mu(\varrho)$ is the unique root of $p^{(1)}\left(\mu ; \lambda_{0}\right)=\varrho$. Furthermore, the derivative of $f\left(\varrho ; \lambda_{0}\right)$ with respect to $\varrho$ is given by

$$
f^{\prime}\left(\varrho ; \lambda_{0}\right)= \begin{cases}\mu(\varrho), & 0 \leqq \varrho \leqq \varrho_{n}, \\ \lambda_{0}, & \varrho>\varrho_{n} .\end{cases}
$$

\section{Summary of Results}

In this section we summarize our principal results and comment on their significance. In Sect. 4, we prove the existence of the pressure in the HYL-model, settling doubts expressed by van Hove [11] about the model's stability. The conditions on the single-particle spectrum which we assume are almost the same as those under which we proved the existence of the free-gas pressure in [7].

In Sect. 5, we solve the variational problem (1.6) to get the following alternative expression for the pressure:

$$
p^{\mathrm{HYL}}(\mu)= \begin{cases}\inf _{\alpha<0}\left\{(\mu-\alpha)^{2} / 4 a+p(\alpha)\right\}, & \mu \leqq \mu^{*}, \\ \sup _{\alpha<0}\left\{(\mu-\alpha)^{2} / 4 a-\alpha^{2} / 2 a+p(\alpha)\right\}, & \mu \geqq \mu^{*},\end{cases}
$$

where $\mu^{*}$ is the unique value of $\mu$ for which the two expressions on the right-hand side of (2.1) are equal; $p(\alpha)$ is the free-gas pressure. Using this expression, we prove in Sect. 6 that the total amount of condensate at chemical potential $\mu$ is given by

$$
\Delta^{\mathrm{HYL}}(\mu)= \begin{cases}-\alpha(\mu) / a, & \mu>\mu^{*}, \\ 0, & \mu<\mu^{*},\end{cases}
$$

where $\alpha(\mu)$ is the unique value of $\alpha$ at which $\sup _{\alpha<0}\left\{(\mu-\alpha)^{2} / 4 a-\alpha^{2} / 2 a+p(\alpha)\right\}$ is attained. A rough calculation of the energy-entropy balance in this model led Thouless [4] to predict a jump in the total amount of condensate as a function of the chemical potential. A calculation by Critchley [12] using a variational principle based on a restricted class of states found further evidence for such a jump. We show that in the case $2 a p^{\prime \prime}(0) \leqq 1$ we have

$$
\lim _{\mu \downarrow \mu^{*}} A^{\mathrm{HYL}}(\mu)=0 .
$$

So that there is no jump; however, in the case $2 a p^{\prime \prime}(0)>1$ we have

$$
\lim _{\mu \downarrow \mu^{*}} \Delta^{\mathrm{HYL}}(\mu)=-\alpha^{*} / a \geqq-\alpha_{t} / a>0,
$$

where $\alpha_{t}$ is the unique root of $2 a p^{\prime \prime}(\alpha)=1$, so that there is a jump in $\Lambda^{\mathrm{HYL}}(\mu)$ at $\mu^{*}$ and the prediction of Thouless (p. 156 of [4]) is confirmed. 
It is interesting to consider the total amount of condensate at fixed meandensity $\varrho$. We can identify $\alpha(\mu)$ as the lowest root of the equation

$$
p^{\prime}(\alpha)=\varrho+\alpha(\mu) / a, \quad \alpha>0 ;
$$

for fixed $\varrho, \alpha(\mu)$ decreases as $a$ increases since $\alpha \mapsto p^{\prime}(\alpha)$ is increasing; hence $-\alpha(\mu) / a$ increases with increasing $a$ so that the total amount of condensate at fixed mean density is an increasing function of the strength of the repulsive interaction. This supports London's conjecture [2] that spatial repulsion between bosons enhances phase-space condensation.

We recall that in the mean-field model [10] the total amount of condensate is given by

$$
\Lambda^{\mathrm{MF}}(\mu)=\left(\varrho(\mu)-\varrho_{c}\right)^{+},
$$

where $\varrho(\mu)$ is the mean-density at chemical potential $\mu$. In this model, there is no jump in $\Delta^{\mathrm{MF}}(\mu)$; moreover, at fixed mean-density it is independent of the strength of the repulsive interaction and equal to the total amount of condensate in the free boson gas at the same mean-density.

The difference in behaviour of the condensate in the two models reflects the difference in origin of the phase-transition. In the mean-field model, as in the free boson gas, condensation is a consequence of the balance between entropy and kinetic energy; in the HYL-model with $2 a p^{\prime \prime}(0)>1$, condensation is a result of the balance between entropy and interaction energy. This difference has a further consequence: in the mean-field model, condensation occurs if and only if it occurs in the corresponding free-gas ( $\varrho_{c}$ finite); in the HYL-model, there is always condensation for $\varrho$ sufficiently large, even when $\varrho_{c}$ is infinite in the corresponding free boson gas. It is to be expected that inclusion of off-diagonal terms in the model hamiltonian would lead to depletion of the condensate; effort expended in solving this difficult problem is likely to prove very rewarding.

\section{The Probabilistic Setting}

In the models considered in this paper, the hamiltonians are diagonal in the occupation number operators; it follows that it is possible to regard the occupation numbers as random variables rather than as operators. We shall do this because it enables us to adopt a powerful method from probability theory.

The probability space on which we define our random variables is the countable set $\Omega$ of terminating sequences of non-negative integers: an element $\omega$ of $\Omega$ is a sequence $\{\omega(j) \in \mathbb{N}: j=1,2, \ldots\}$ satisfying $\sum_{j \geqq 1} \omega(j)<\infty$. The basic random variables are the occupation numbers $\left\{\sigma_{j}: j=1,2, \ldots\right\}$; they are the evaluation maps $\sigma_{j}: \Omega \rightarrow \mathbb{N}$ defined by $\sigma_{j}(\omega)=\omega(j)$ for each $\omega$ in $\Omega$. The total number of particles $N(\omega)$ in the configuration $\omega$ is defined by

$$
N(\omega)=\sum_{j \geqq 1} \sigma_{j}(\omega) .
$$

Motivated by the discussion in Sect. 1 , we define, for each integer $l \geqq 1$, the free-gas hamiltonian $H_{l}$ by

$$
H_{l}(\omega)=\sum_{j \geqq 1} \lambda_{l}(j) \sigma_{j}(\omega)
$$


and the mean-field hamiltonian $H_{l}^{\mathrm{MF}}$ by

$$
H_{l}^{\mathrm{MF}}(\omega)=H_{l}(\omega)+\frac{a}{2 V_{l}} N(\omega)^{2},
$$

where $a$ is a strictly positive real number. Since $\Omega$ is a countable set, we may specify a probability measure on $\Omega$ by giving its value at each point of $\omega$ of $\Omega$. The free-gas grand canonical measure $\mathbb{P}_{l}^{\mu}[]$ is defined by

$$
\mathbb{P}_{l}^{\mu}[\omega]=e^{\beta\left\{\mu N(\omega)-H_{l}(\omega)-V_{l} p_{l}(\mu)\right\}} .
$$

Here $p_{l}(\mu)$ is the free-gas pressure given in terms of the $\lambda_{l}(j)$ by $(1.11)$; it satisfies

$$
e^{\beta V_{l} p_{l}(\mu)}=\sum_{\omega \in \Omega} e^{\beta\left\{\mu N(\omega)-H_{l}(\omega)\right\}} .
$$

The large deviation method which we are going to employ in proving the existence of the pressure in the HYL-model is illustrated simply in the case of the mean-field model. The mean-field pressure $p_{l}^{\mathrm{MF}}(\mu)$ satisfies

$$
e^{\beta V_{l} p_{l}^{\mathrm{MF}}(\mu)}=\sum_{\omega \in \Omega} e^{\beta\left\{\mu N(\omega)-H_{l}^{\mathrm{MF}}(\omega)\right\}} .
$$

Introducing the particle number-density $X_{l}=N / V_{l}$, we can re-write (3.6) as

$$
e^{\beta V_{l} p_{l}^{\mathrm{MF}}(\mu)}=\sum_{\omega \in \Omega} e^{\beta V_{l}\left(u \circ X_{l}\right)(\omega)} e^{\beta\left\{\mu N(\omega)-H_{l}(\omega)\right\}},
$$

where $u(x)=-a x^{2} / 2$; using (3.5), we have

$$
e^{\beta V_{l} p_{l}^{\mathrm{MF}}(\mu)}=e^{\beta V_{l} p_{l}(\mu)} \sum_{\omega \in \Omega} e^{\beta V_{l}\left(u \circ X_{l}\right)(\omega)} \mathbb{P}_{l}^{\mu}[\omega] .
$$

Introducing the probability measure $\mathbb{K}_{l}^{\mu}$ on $[0, \infty)$ by

$$
\mathbb{K}_{l}^{\mu}=\mathbb{P}_{l}^{\mu} \circ X_{l}^{-1}
$$

we can re-write (3.8) as

$$
e^{\beta V_{l} p_{l}^{\mathrm{MF}}}(\mu)=e^{\beta V_{l} p_{l}(\mu)} \int_{[0, \infty)} e^{\beta V_{l} u(x)} \mathbb{K}_{l}^{\mu}[d x],
$$

so that

$$
p_{l}^{\mathrm{MF}}(\mu)=p_{l}(\mu)+\frac{1}{\beta V_{l}} \ln \int_{[0, \infty)} e^{\beta V_{l} u(x)} \mathbb{K}_{l}^{\mu}[d x] .
$$

This expression for $p_{l}^{\mathrm{MF}}(\mu)$ suggests the use of Laplace's method to complete the proof that $p^{\mathrm{MF}}(\mu)=\lim _{l \rightarrow \infty} p_{l}^{\mathrm{MF}}(\mu)$ exists. Varadhan's theorem [6] provides an efficient way of doing this; the conditions on the sequence $\left\{\mathbb{K}_{l}^{\mu}: l=1,2, \ldots\right\}$ are stated abstractly:

Let $E$ be a regular topological space and $\left\{V_{l}: l=1,2, \ldots\right\}$ a sequence of positive numbers diverging to $+\infty$. Let $\left\{\mathbb{K}_{l}: l=1,2, \ldots\right\}$ be a sequence of probability measures on the Borel subsets of $E$. We say that $\left\{\mathbb{K}_{l}\right\}$ obeys the large deviation principle with constants $\left\{V_{l}\right\}$ and rate-function $I: E \rightarrow[0, \infty]$ if the following conditions are satisfied: 
(LD1) I( $\cdot)$ is lower semi-continuous.

(LD2) For each $m<\infty$ the set $\{x: I(x) \leqq m\}$ is compact.

(LD3) For each closed set $C, \lim _{l \rightarrow \infty} \sup \frac{1}{V_{l}} \ln \mathbb{K}_{l}[C] \leqq-\inf _{C} I(x)$.

(LD4) For each open set $G, \lim _{l \rightarrow \infty} \inf \frac{1}{V_{l}} \ln \mathbb{K}_{l}[G] \geqq-\inf _{G} I(x)$.

We are now ready to state

Varadhan's Theorem [6]. Let $\left\{\mathbb{K}_{l}\right\}$ be a sequence of probability measures on the Borel subsets of E satisfying the large deviation principle with constant $\left\{V_{l}\right\}$ and ratefunction $I(\cdot)$. Then, for any continuous function $G: E \rightarrow \mathbb{R}$ which is bounded above, we have

$$
\lim _{l \rightarrow \infty} \frac{1}{V_{l}} \ln \int_{E} e^{V_{l} G(x)} \mathbb{K}_{l}[d x]=\sup _{E}\{G(x)-I(x)\} .
$$

Returning to the proof of the existence of the pressure in the mean-field model, we make use of a large deviation result for the free-gas measures $\left\{\mathbb{K}_{l}^{\mu}\right\}$ defined at (3.9); the proof is given in the Appendix:

Theorem A1. Suppose that (S1) and (S2) hold; then for each $\mu<0$ the sequence $\left\{\mathbb{K}_{l}^{\mu}: l=1,2, \ldots\right\}$ of probability measures on $[0, \infty)$ satisfies the large-deviation principle with constants $\left\{V_{l}\right\}$ and rate-function

$$
I^{\mu}(x)=p(\mu)+f(x)-\mu x .
$$

Applying Theorem A1 and Varadhan's theorem, we have

$$
\begin{aligned}
\lim _{l \rightarrow \infty} \frac{1}{\beta V_{l}} \ln \int_{[0, \infty)} e^{\beta V l u(x)} \mathbb{K}_{l}^{\mu}[d x] & =\sup _{[0, \infty)}\left\{u(x)-I^{\mu}(x)\right\} \\
& =-p(\mu)+\sup _{[0, \infty)}\left\{\mu x-f(x)-\frac{a x^{2}}{2}\right\} .
\end{aligned}
$$

Combining this with the conclusion of Proposition 1, (3.11) yields the existence of the limit $p^{\mathrm{MF}}(\mu)=\lim _{l \rightarrow \infty} p^{\mathrm{MF}}(\mu)$ and the following formula:

$$
p^{\mathrm{MF}}(\mu)=\sup _{[0, \infty)}\left\{\mu x-f(x)-\frac{a x^{2}}{2}\right\} .
$$

So far this has been proved only for $\mu<0$; nevertheless, the pressure $p^{\mathrm{MF}}(\mu)$ exists for all real $\mu$ and is given by (3.15). To prove this, we use a device which we will need later. Fix $\alpha<0$; then a straightforward manipulation yields

$$
e^{\beta V_{l} p_{l}^{\mathrm{MF}}(\mu)}=e^{\beta V_{l} p_{l}(\alpha)} \sum_{\omega \in \Omega} e^{\beta V_{l}\left(u_{\alpha^{\circ}} X_{l}\right)(\omega)} \mathbb{P}_{l}^{\alpha}[\omega],
$$

where $u_{\alpha}(x)=(\mu-\alpha) x-a x^{2} / 2$, so that

$$
p_{l}^{\mathrm{MF}}(\mu)=p_{l}(\alpha)+\frac{1}{\beta V_{l}} \ln \int_{[0, \infty)} e^{\beta V_{l}\left(u_{\alpha} \circ X_{l}\right)(x)} \mathbb{K}_{l}^{\alpha}[d x] .
$$


Proceeding as before, we get (3.15) for all real $\mu$.

A simple exercise yields the following alternative expression for $p^{\mathrm{MF}}(\mu)$ :

$$
p^{\mathrm{MF}}(\mu)=\inf _{\alpha<0}\left\{\frac{(\mu-\alpha)^{2}}{2 a}+p(\alpha)\right\} \text {. }
$$

This result was obtained earlier [10] by other means; in that proof, the subleties arising from the first-order phase-transition in the free-boson gas were all too evident; in the proof we have just given, they are tidied away in the verification of the large deviation principle for the measures $\left\{\mathbb{K}_{l}^{\alpha}: l=1,2, \ldots\right\}$.

\section{The Existence of the Pressure in the HYL-Model}

In this section, we obtain the main results of this paper: we prove the existence of the pressure in the HYL-model, and we derive an expression for the pressure as a function of the chemical potential. Motivated by the discussion in Sect. 1, we define, for each integer $l \geqq 1$, the HYL-hamiltonian $H_{l}^{\mathrm{HYL}}$ by

$$
H_{l}^{H Y L}(\omega)=H_{l}^{0}(\omega)+\frac{a}{2 V_{l}}\left\{2 N(\omega)^{2}-\sum_{j=1}^{m_{l}} \sigma_{j}(\omega)^{2}\right\}
$$

for $a>0$ and $\left\{m_{l}: l=1,2, \ldots\right\}$ a sequence of positive integers such that $m_{l} / V_{l} \rightarrow 0$ as $l \rightarrow \infty$. Recall that $\lambda_{0}$ was defined to be $\inf \{\lambda: F(\lambda)>0\}$.

Theorem 1. Suppose that (S1) and (S2) hold and that $\lambda_{0}$ is a point of continuity of $F$; then the pressure

$$
p^{\mathrm{HYL}}(\mu)=\lim _{l \rightarrow \infty} p_{l}^{\mathrm{HYL}}(\mu)
$$

exists for all real values of $\mu$ and is given by

$$
p^{H Y L}(\mu)=\sup _{0 \leqq x \leqq x_{0}}\left\{\mu x_{0}-f\left(x_{0}-x_{1} ; \lambda_{0}\right)-\frac{a}{2}\left(2 x_{0}^{2}-x_{1}^{2}\right)\right\} .
$$

The proof makes use of Varadhan's theorem and a large deviation results for the joint distribution of $\sigma_{1}$ and $\sum_{j>1} \sigma_{j}$. Define the vector-valued random variable
$X_{l}: \Omega \rightarrow \mathbb{R}^{2}$ by

$$
X_{l}^{(1)}(\omega)=V_{l}^{-1}(\omega), \quad X_{l}^{(2)}(\omega)=V_{l}^{-1} \sum_{j>1} \sigma_{j}(\omega),
$$

and, for $\mu<0$, define the sequence $\left\{\mathbb{K}_{l}^{\mu}: l=1,2, \ldots\right\}$ of probability measures on $\mathbb{R}_{+}^{2}$ by

$$
\mathbb{K}_{l}^{\mu}=\mathbb{P}_{l}^{\mu} \circ X_{l}^{-1}
$$

In the Appendix, we prove

Theorem A2. Suppose that (S1) and (S2) hold and that $\lambda(2)=\lim _{l \rightarrow \infty} \lambda_{l}(2)$ exists and is a point of continuity of $F$. Then, for each $\mu<0$, the sequence $\left\{\mathbb{K}_{l}^{\mu}: l=1,2, \ldots\right\}$ satisfies 
the large deviation principle with constants $\left\{V_{l}\right\}$ and rate-function $I^{\mu}(\cdot)$ given by

$$
I^{\mu}(x)=p(\mu)+f\left(x_{2} ; \lambda(2)\right)-\mu\left(x_{1}+x_{2}\right), \quad x_{1} \geqq 0, \quad x_{2} \geqq 0 .
$$

Proof of Theorem. Define $\bar{H}_{l}(\omega)$ by

$$
\bar{H}_{l}(\omega)=\sum_{j \geqq 1} \bar{\lambda}_{l}(j) \sigma_{j}(\omega)+\frac{a}{2 V_{l}}\left\{2 N(\omega)^{2}-\sigma_{1}(\omega)^{2}\right\},
$$

where

$$
\bar{\lambda}_{l}(j)= \begin{cases}0, & j=1, \\ \max \left\{\lambda_{0}, \lambda_{l}(j)\right\}, & j \geqq 2,\end{cases}
$$

so that

$$
\lim _{l \rightarrow \infty} \bar{\lambda}_{l}(2)=\lambda_{0} \quad \text { and } \quad \bar{H}_{l}(\omega) \geqq H_{l}^{\mathrm{HYL}}(\omega) .
$$

It is easy to check that (S1) and (S2) hold for the new double sequence $\left\{\bar{\lambda}_{l}(j)\right\}$ and that the integrated density of states is unchanged. Let $\bar{p}_{l}(\mu)$ be the grand canonical pressure corresponding to the hamiltonian $\bar{H}_{l}$; an application of Varadhan's theorem together with Theorem A2 yields

$$
\begin{aligned}
\bar{p}(\mu) & =\lim _{l \rightarrow \infty} \bar{p}_{l}(\mu) \\
& =\sup _{x_{1} \geqq 0, x_{2} \geqq 0}\left\{\mu\left(x_{1}+x_{2}\right)-f\left(x_{2} ; \lambda_{0}\right)-\frac{a}{2}\left[2\left(x_{1}+x_{2}\right)^{2}-x_{1}^{2}\right]\right\} \\
& =\sup _{0 \leqq x_{1} \leqq x_{0}}\left\{\mu x_{0}-f\left(x_{0}-x_{1} ; \lambda_{0}\right)-\frac{a}{2}\left[2 x_{0}^{2}-x_{1}^{2}\right]\right\} .
\end{aligned}
$$

Since $\bar{H}_{l}(\omega) \geqq H_{l}^{\mathrm{HYL}}(\omega)$ we have $\bar{p}_{l}(\mu) \leqq p_{l}^{\mathrm{HYL}}(\mu)$ so that

$$
\bar{p}(\mu) \leqq \liminf _{l \rightarrow \infty} p_{l}^{\mathrm{HYL}}(\mu)
$$

The proof of the upper bound to the pressure is less straightforward and makes use of the following entropy estimate:

Lemma 1. For $n=0,1,2, \ldots$ and $k=1,2, \ldots$

$$
\left(\begin{array}{c}
n+k-1 \\
n
\end{array}\right) \leqq e^{4(n k)^{1 / 2}} .
$$

Proof. The proof is by induction on $k$. Evidently (4.9) holds for $k=1$. Suppose that (4.9) holds for all $k^{\prime}$ less than or equal to some fixed $k$; then

$$
\begin{aligned}
\left(\begin{array}{c}
n+k \\
n
\end{array}\right) & =\frac{n+k}{k}\left(\begin{array}{c}
n+k-1 \\
n
\end{array}\right) \\
& \leqq\left(1+\frac{n}{k}\right) e^{4(n k)^{1 / 2}}
\end{aligned}
$$


But

Hence

$$
\begin{aligned}
e^{4 n^{1 / 2}\left\{(k+1)^{1 / 2}-k^{1 / 2}\right\}} & =e^{4 n^{1 / 2} /\left\{(k+1)^{1 / 2}+k^{1 / 2}\right\}} \\
& \geqq e^{2 n^{1 / 2} /(k+1)^{1 / 2}} \geqq e^{(2 n / k)^{1 / 2}} \\
& \geqq 1+\left(\frac{2 n}{k}\right)^{1 / 2}+\frac{1}{2}\left(\frac{2 n}{k}\right) \geqq 1+\frac{n}{k}
\end{aligned}
$$

$$
\left(\begin{array}{c}
n+k \\
n
\end{array}\right) \leqq e^{4 n^{1 / 2}(k+1)^{1 / 2}}
$$

Returning to the proof of Theorem 1 , define $k_{l}=\max \left\{m_{l}, V_{l} F_{l}\left(\lambda_{0}\right)\right\}$ and put

and

$$
\underline{\lambda}_{l}(j)= \begin{cases}0, & j=1, \ldots, k_{l}, \\ \lambda_{l}(j), & j \geqq k_{l}+1,\end{cases}
$$

$$
\underline{H}_{l}(\omega)=\sum_{j \geqq 1} \lambda_{l} \sigma_{j}(\omega)+\frac{a}{2 V_{l}}\left\{2 N(\omega)^{2}-\left(\sum_{j=1}^{k_{l}} \sigma_{j}(\omega)\right)^{2}\right\} ;
$$

since $\underline{\lambda}_{l}(j) \leqq \lambda_{l}(j)$ for all $j$ and

$$
\left(\sum_{j=1}^{k_{l}} \sigma_{j}(\omega)\right)^{2} \geqq\left(\sum_{j=1}^{m_{l}} \sigma_{j}(\omega)\right)^{2} \geqq \sum_{j=1}^{m_{l}} \sigma_{j}(\omega)^{2},
$$

we have $\underline{H}_{l}(\omega) \leqq H_{l}^{\mathrm{HYL}}(\omega)$ and $\underline{p}_{l}(\mu) \geqq p_{l}^{\mathrm{HYL}}(\mu)$, where $\underline{p}_{l}(\mu)$ is the grand canonical pressure corresponding to the hamiltonian $\underline{H}_{l}$.

Now

$$
\begin{aligned}
e^{\beta V_{l} \underline{\underline{p}}_{l}(\mu)} & =\sum_{\omega \in \Omega} e^{\beta\left\{\mu N(\omega)-H_{l}(\omega)\right\}} \\
& =\sum_{n_{1}, n_{2}, \ldots} e^{\beta\left\{\sum_{J \supseteq 1}\left(\mu-\lambda_{l}(j)\right) n_{J}-\frac{a}{2 V_{l}}\left[2\left(\sum_{J \geqq 1} n_{J}\right)^{2}-\left(\sum_{J=1}^{k_{l}} n_{J}\right)^{2}\right]\right\}} \\
& =\sum_{m=0}^{\infty}\left(\begin{array}{c}
m+k_{l}-1 \\
m
\end{array}\right) Z_{l}(m),
\end{aligned}
$$

where

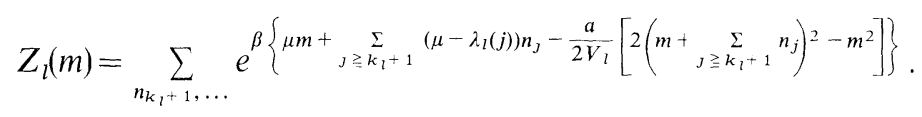

By Lemma 1, we have

$$
e^{\beta V_{l} \underline{p}(\mu)} \leqq \sum_{m=0}^{\infty} e^{4\left(m k_{l}\right)^{1 / 2}} Z_{l}(m)
$$

put

$$
\begin{aligned}
\underline{\underline{H}}_{l}(\omega)= & \sum_{j \geqq 1} \underline{\lambda}_{l}(j) \sigma_{j}(\omega)+\frac{a}{2 V_{l}}\left\{2\left(\sum_{j \geqq 1} \sigma_{j}(\omega)^{2}\right)-\sigma_{1}(\omega)^{2}\right\} \\
& -\frac{4}{\beta}\left(k_{l} \sigma_{1}(\omega)\right)^{1 / 2}
\end{aligned}
$$


where

$$
\varliminf_{l}(j)= \begin{cases}0, & j=1, \\ \lambda_{l}\left(k_{l}+j-1\right), & j \geqq 2,\end{cases}
$$

and let $\underline{\underline{p}}_{l}(\mu)$ be the grand canonical pressure corresponding to the hamiltonian $\stackrel{H}{H}_{l}$. It follows from the definitions that

$$
e^{\beta V_{l} \underline{\underline{\underline{p}}} l(\mu)}=\sum_{m=0}^{\infty} e^{4\left(m k_{l}\right)^{1 / 2}} Z_{l}(m) .
$$

Hence, using (4.13), we have

$$
\underline{\underline{p}}_{l}(\mu) \geqq \underline{p}_{l}(\mu) \geqq p_{l}^{\mathrm{HYL}}(\mu) .
$$

It is straightforward to show that the new double sequence $\left\{\lambda_{l}(j)\right\}$ satisfies (S1) and (S2) with the integrated density of states unchanged, and that $\lim _{l \rightarrow \infty} \lambda_{l}(2)=\lambda_{0}$. To complete the proof of Theorem 1, we use Theorem A2 together with the following version of Varadhan's theorem which can be deduced from Sect. 3 of [6] when $E$ is a complete separable metric space:

Let $\left\{G_{l}: l=1,2, \ldots\right\}$ be a sequence of continuous functions uniformly bounded above which converges uniformly on bounded subsets to $G$. Let $\left\{\mathbb{K}_{l}: l=1,2, \ldots\right\}$ be a sequence of probability measures which satisfies the large deviation principle with constants $\left\{V_{l}\right\}$ and rate-function $I(\cdot)$. Then

$$
\lim _{l \rightarrow \infty} \frac{1}{V_{l}} \ln \int_{E} e^{V_{l} G_{l}(x)} K_{l}[d x]=\sup _{E}\{G(x)-I(x)\} .
$$

Applying this with $G_{l}(x)$ defined by

$$
G_{l}(x)=(\mu-\alpha)\left(x_{1}+x_{2}\right)-\frac{a}{2}\left[2\left(x_{1}+x_{2}\right)^{2}-x_{1}^{2}\right]-\frac{4}{\beta}\left(\frac{k_{l}}{V_{l}}\right)^{1 / 2} x_{1}^{1 / 2},
$$

we have

$$
\begin{aligned}
\underline{\underline{p}}(\mu) & =\lim _{l \rightarrow \infty} \underline{\underline{p}}_{l}(\dot{\mu}) \\
& =\sup _{x_{1} \geqq 0, x_{2} \geqq 0}\left\{\mu\left(x_{1}+x_{2}\right)-f\left(x_{2} ; \lambda_{0}\right)-\frac{\lambda}{2}\left[2\left(x_{1}+x_{2}\right)^{2}-x_{1}^{2}\right]\right\} \\
& =\sup _{0 \leqq x_{1} \leqq x_{0}}\left\{\mu x_{0}-f\left(x_{0}-x_{1} ; \lambda_{0}\right)-\frac{a}{2}\left[2 x_{0}^{2}-x_{1}^{2}\right]\right\} \\
& =\bar{p}(\mu), \text { by (4.7). }
\end{aligned}
$$

Since $\underline{p}_{l}(\mu) \geqq p_{l}^{\mathrm{HYL}}(\mu)$, we have

$$
\underline{\underline{p}}(\mu) \geqq \lim _{l \rightarrow \infty} \sup _{l}^{\mathrm{HYL}}(\mu) .
$$

Thus we have, using (4.8),

$$
\underline{p}_{l}(\mu) \geqq \lim _{l \rightarrow \infty} \sup p_{l}^{\mathrm{HYL}}(\mu) \geqq \liminf _{l \rightarrow \infty} p_{l}^{\mathrm{HYL}}(\mu) \geqq \bar{p}(\mu),
$$

so that, by (4.19), the limit $p^{\mathrm{HYL}}(\mu)=\lim _{l \rightarrow \infty} p_{l}^{\mathrm{HYL}}(\mu)$ exists and is given by (4.2). 


\subsection{The Grand Canonical Pressure}

In this section, we solve the variational problem which arises when we try to express the pressure $p^{\mathrm{HYL}}(\mu)$, given by (4.17), in terms of the free-gas pressure $p(\alpha)$, $\alpha<0$. We prove the following theorem:

Theorem 2. Let $\mu_{t}=\inf _{\alpha<0}\left\{2 a p^{\prime}(\alpha)-\alpha\right\}$ and let $\mu_{c}=2 a \varrho_{c}$; then there exists a unique value $\mu^{*}$ of $\mu$ in the interval $\left(\mu_{t}, \mu_{c}\right)$ such that

$$
p^{\mathrm{HYL}}(\mu)= \begin{cases}\inf _{\alpha<0}\left\{(\mu-\alpha)^{2} / 4 a+p(\alpha)\right\}, & \mu \leqq \mu^{*}, \\ \sup _{\alpha<0}\left\{(\mu-\alpha)^{2} / 4 a-\alpha^{2} / 2 a+p(\alpha)\right\}, & \mu \geqq \mu^{*} .\end{cases}
$$

Remark. The point $\mu^{*}$ is the unique value of $\mu$ for which the two expressions on the right-hand side of (5.1) become equal.

Proof. Put

and

$$
g(x)= \begin{cases}\mu^{2} / 2 a+a x^{2}-\mu x, & 0 \leqq x \leqq \mu / 2 a \\ -a x^{2}+\mu x, & x>\mu / 2 a\end{cases}
$$

$$
f_{0}(x)=f\left(x ; \lambda_{0}\right) .
$$

Taking the supremum over the set $\left\{x_{1} \geqq 0\right\}$ and replacing $x_{2}$ by $x$, we have

$$
\begin{aligned}
p^{\mathrm{HYL}}(\mu) & =\sup _{x_{1} \geqq 0, x_{2} \geqq 0}\left\{-\frac{a}{2}\left[2\left(x_{1}+x_{2}\right)^{2}-x_{1}^{2}\right]+\mu\left(x_{1}+x_{2}\right)-f_{0}\left(x_{2}\right)\right\} \\
& =\sup _{x \geqq 0}\left\{g(x)-f_{0}(x)\right\} .
\end{aligned}
$$

The functions $f_{0}$ and $g$ are differentiable on $(0, \infty)$; on $(0, \infty), g^{\prime}$ is non-positive while $f_{0}^{\prime}$ is non-positive on $\left(0, \varrho_{c}\right]$ and non-negative on $\left[\varrho_{c}, \infty\right)$, so that the equation $g^{\prime}(x)-f_{0}^{\prime}(x)=0$ can only be satisfied in $\left(0, \varrho_{c}\right]$. Since $g(x)-f_{0}(x)$ decreases to $-\infty$ as $x \rightarrow \infty$, the supremum of $g-f_{0}$ is attained either at zero or at a stationary point in $\left(0, \varrho_{c}\right]$; however, $f_{0}^{\prime}(x)$ decreases to $-\infty$ as $x \downarrow 0$ while $g^{\prime}(x)$ remains finite so that the supremum cannot be attained at zero. We have

$$
p^{\mathrm{HYL}}(\mu)=\sup _{\left(0 . \varrho_{c}\right]}\left\{g(x)-f_{0}(x)\right\}=g\left(x_{0}\right)-f_{0}\left(x_{0}\right),
$$

where $x_{0}$ is a point of $\left(0, \varrho_{c}\right]$ at which $f_{0}^{\prime}(x)=g^{\prime}(x)$. We must distinguish two cases:

(i) For $\mu>2 a \varrho_{c}$ we have $g(x)=\mu^{2} / 2 a+a x^{2}-\mu x, x \in\left(0, \varrho_{c}\right]$, so that a stationary point is a root of the equation

$$
f_{0}^{\prime}(x)=2 a x-\mu .
$$

Since $\varrho_{c} \leqq \varrho_{n}$, we have $f_{0}^{\prime}(x)=\alpha(x) \leqq 0$ where $\alpha(x)$ is the unique real root of $p^{\prime}(\alpha)=x$. Putting $h(\alpha)=2 a p^{\prime}(\alpha)-\alpha$, Eq. (5.6) becomes $h(\alpha(x))=\mu$. Now $\alpha \mapsto h(\alpha), \alpha \leqq 0$, is convex; since $h(\alpha)$ increases to $+\infty$ as $\alpha \rightarrow-\infty$ and $h(0)=2 a \varrho_{c}<\mu$, the equation, $h(\alpha)=\mu$ has a unique solution on $(-\infty, 0]$. Thus, for $\mu>2 a \varrho_{c}$,

$$
p^{\mathrm{HYL}}(\mu)=\mu^{2} / 2 a+a\left(p^{\prime}\left(\alpha_{1}\right)\right)^{2}-\left(\mu+\alpha_{1}\right) p\left(\alpha_{1}\right)+p\left(\alpha_{1}\right),
$$

where $\alpha_{1} \leqq 0$ is the unique root of $2 a p^{\prime}(\alpha)-\alpha=\mu$. 
(ii) For $\mu \leqq 2 a \varrho_{c}$, we define $\mu_{t}$ by $\mu_{t}=\inf _{\alpha<0} h(\alpha)$; this occurs at the unique point $\alpha_{t}$ [because $\alpha \mapsto h(\alpha)$ is strictly convex] where

$$
\alpha_{t}= \begin{cases}0, & 2 a p^{\prime \prime}(0) \leqq 1, \\ \text { the unique real root of } 2 a p^{\prime \prime}(\alpha)=1, & \text { otherwise }\end{cases}
$$

Note that $\mu_{t} \leqq h(0)=2 a \varrho_{c}$. For $\mu<\mu_{t}$, the equation $h(\alpha)=\mu$ has no solution in $(-\infty, 0]$ so that the supremum of $g-f_{0}$ must be attained on $\left[\mu / 2 a, \varrho_{c}\right]$. At a stationary point we have

$$
2 a x+f_{0}^{\prime}(x)=\mu
$$

or, equivalently,

$$
k(\alpha)=\mu, \quad \alpha \leqq 0,
$$

where

$$
k(\alpha)=2 a p^{\prime}(\alpha)+\alpha .
$$

Since $\alpha \mapsto k(\alpha)$ is strictly increasing from $-\infty$ to $k(0)=2 a \varrho_{c}>\mu_{t}$, the equation $k(\alpha)=\mu$ has a unique solution $\alpha_{2}$ and $x_{2}=p^{\prime}\left(\alpha_{2}\right)$ must lie in $\left[\mu / 2 a, \varrho_{c}\right]$. Thus, for $\mu \leqq \mu_{t}$,

$$
p^{\mathrm{HYL}}(\mu)=\left(\mu-\alpha_{2}\right)^{2} / 4 a+p\left(\alpha_{2}\right)-\inf _{\alpha<0}\left\{\frac{(\mu-\alpha)^{2}}{4 a}+p(\alpha)\right\} .
$$

When $2 a p^{\prime \prime}(0) \leqq 1$, we have $\mu_{t}=2 a \varrho_{c}$ and so we have exhausted all cases; we conclude that $p^{\mathrm{HYL}}(\mu)$ is given by

$$
p^{\mathrm{HYL}}(\mu)= \begin{cases}\inf _{x<0}\left\{\frac{(\mu-\alpha)^{2}}{4 a}+p(\alpha)\right\}, & \mu \leqq 2 a \varrho_{c}, \\ \sup _{\alpha<0}\left\{\frac{(\mu-\alpha)^{2}}{4 a}-\frac{\alpha^{2}}{2 a}+p(\alpha)\right\}, & \mu>2 a \varrho_{c} .\end{cases}
$$

When $2 a p^{\prime \prime}(0)>1$, it remains to consider the case in which $\mu$ lies in the interval $\left(\mu_{t}, 2 a \varrho_{c}\right]$. In this case, the equation $h(\alpha)=\mu$ has two solutions $\alpha^{\prime}, \alpha^{\prime \prime}$ with $\alpha^{\prime}<\alpha^{\prime \prime}$; $h(\alpha)-\mu$ is positive for $\alpha$ in $\left(-\infty, \alpha^{\prime}\right)$ and $\left(\alpha^{\prime \prime}, 0\right)$ and is negative for $\alpha$ in $\left(\alpha^{\prime}, \alpha^{\prime \prime}\right)$ so that there is a local maximum at $\alpha^{\prime}$ and a local minimum at $\alpha^{\prime \prime}$. Notice that $x^{\prime}=p^{\prime}\left(\alpha^{\prime}\right)$ $\leqq \frac{\mu}{2 a}$ since $2 a p^{\prime}\left(\alpha^{\prime}\right)=\mu+\alpha^{\prime} \leqq \mu$. On the other hand, the equation $k(\alpha)=\mu$ has a unique solution $\alpha^{\prime \prime \prime}$; since $k^{\prime}(\alpha)>0$, there is a local maximum at $\alpha^{\prime \prime \prime}=p^{\prime}\left(\alpha^{\prime \prime \prime}\right)>\mu / 2 a$. Put

$$
p_{1}(\mu)=\left(\mu-\alpha^{\prime}\right)^{2} / 4 a-\alpha^{\prime 2} / 2 a+p\left(\alpha^{\prime}\right)
$$

and

$$
p_{2}(\mu)=\left(\mu-\alpha^{\prime \prime \prime}\right)^{2} / 4 a+p\left(\alpha^{\prime \prime \prime}\right)
$$

then

$$
p^{\mathrm{HYL}}(\mu)=\max \left\{p_{1}(\mu), p_{2}(\mu)\right\} .
$$


We shall prove that, for $\mu_{t}<2 a \varrho_{c}$, there is a unique value $\mu^{*}$ of $\mu$ for which $p_{1}(\mu)=p_{2}(\mu)$ and that

$$
p^{\mathrm{HYL}}(\mu)= \begin{cases}p_{2}(\mu), & \mu_{t} \leqq \mu \leqq \mu^{*} \\ p_{1}(\mu), & \mu^{*} \leqq \mu \leqq 2 a \varrho_{c}\end{cases}
$$

Since

$$
2 a p^{\prime}\left(\alpha^{\prime}\right)=\mu+\alpha^{\prime}
$$

and

$$
2 a p^{\prime}\left(\alpha^{\prime \prime \prime}\right)=\mu-\alpha^{\prime \prime \prime}
$$

we have

$$
p^{\prime}\left(\alpha^{\prime}\right)-p^{\prime}\left(\alpha^{\prime \prime \prime}\right)=\left(\alpha^{\prime}+\alpha^{\prime \prime \prime}\right) / 2 a<0
$$

so that $\alpha^{\prime}<\alpha^{\prime \prime \prime}$ since $\alpha \mapsto p^{\prime}(\alpha)$ is strictly increasing. Now

$$
\begin{aligned}
& \frac{d p_{1}}{d \mu}(\mu)=\left(\mu-\alpha^{\prime}\right) / 2 a, \\
& \frac{d p_{2}}{d \mu}(\mu)=\left(\mu-\alpha^{\prime \prime \prime}\right) / 2 a,
\end{aligned}
$$

so that $\frac{d p_{2}}{d \mu}(\mu)<\frac{d p_{1}}{d \mu}(\mu)$, which implies that $\mu \mapsto p_{1}(\mu)-p_{2}(\mu)$ is strictly increasing. Thus it is sufficient to prove that

$$
p_{1}\left(\mu_{t}\right)-p_{2}\left(\mu_{t}\right)<0
$$

and

$$
p_{1}\left(2 a \varrho_{c}\right)-p_{2}\left(2 a \varrho_{c}\right)>0
$$

Let $\delta_{t}$ be the unique value of $\alpha$ for which $k(\alpha)=\mu_{t}$; we know that $\alpha_{t}$ satisfies $h\left(\alpha_{t}\right)=\mu_{t}$ so that, by the above argument, $\alpha_{t}<\delta_{t}$. Thus

$$
\begin{aligned}
p_{l}\left(\mu_{t}\right) & =\left(\mu_{t}-\alpha_{t}\right)^{2} / 4 a-\alpha_{t}^{2} / 2 a+p\left(\alpha_{t}\right) \\
& <\left(\mu_{t}-\delta_{t}\right)^{2} / 4 a-\delta_{t}^{2} / 2 a+p\left(\delta_{t}\right) \\
& <\left(\mu_{t}-\delta_{t}\right)^{2} / 4 a+p\left(\delta_{t}\right)=p_{2}\left(\mu_{t}\right),
\end{aligned}
$$

where we have used the fact that

$$
\alpha \mapsto\left(\mu_{t}-\alpha\right)^{2} / 4 a-\alpha^{2} / 2 a+p(\alpha)
$$

is increasing. Let $\gamma_{t}$ be the unique root of $h(\alpha)=2 a \varrho$ in $(-\infty, 0)$; then

$$
p_{l}\left(2 a \varrho_{c}\right)=\left(2 a \varrho_{c}-\gamma_{t}\right)^{2} / 4 a-\gamma_{t}^{2} / 4 a+p\left(\gamma_{t}\right)>a \varrho_{c}^{2}+p(0)=p_{2}\left(2 a \varrho_{c}\right),
$$

since $\alpha \mapsto\left(2 a \varrho_{c}-\alpha\right)^{2} / 4 a-\alpha^{2} / 2 a+p(\alpha)$ is decreasing. 


\section{The Condensate}

In order to investigate the phase-transition more closely, we compute the total amount of condensate $\Delta(\mu)$. Let $X_{l}(\lambda ; \omega)$ be the random variable defined by

$$
X_{l}(\lambda ; \omega)=V_{l}^{-1} \sum_{\left\{j: \lambda_{l}(j) \leqq \lambda\right\}} \sigma_{j}(\omega)
$$

it presents the density of particles with kinetic energy less than $\lambda$ in the configuration $\omega$. The total amount of condensate $\Delta(\mu)$ is defined by

$$
\Lambda(\mu)=\lim _{\lambda \downarrow 0} \lim _{l \rightarrow \infty} \widetilde{\mathbb{E}}_{l}^{\mu}\left[X_{l}(\lambda)\right]
$$

where $\tilde{\mathbb{E}}_{l}^{\mu}[\cdot]$ denotes the expectation with respect to the grand canonical measure determined by the hamiltonian $\tilde{H}_{l}$ :

$$
\tilde{\mathbb{E}}_{l}^{\mu}[F]=\frac{\sum_{\omega \in \Omega} F(\omega) e^{\beta\left\{\mu N(\omega)-\tilde{H}_{l}(\omega)\right\}}}{\sum_{\omega \in \Omega} e^{\beta\left\{\mu N(\omega)-\tilde{H}_{l}(\omega)\right\}}} .
$$

It is evident that (6.2) is a way of making precise the notion of the "density of particles with zero kinetic energy." To avoid complicating the discussion, we assume in this section that the integrated density of states is continuous.

Theorem 3. In the HYL-model, the total amount of condensate $\Delta(\mu)$ is given by

$$
\Delta(\mu)= \begin{cases}-\alpha(\mu) / a, & \mu>\mu^{*}, \\ 0, & \mu<\mu^{*},\end{cases}
$$

where $\alpha(\mu)$ is the unique value of $\alpha$ at which $\sup _{\alpha<0}\left\{\frac{(\mu-\alpha)^{2}}{4 a}-\frac{\alpha^{2}}{2 a}+p(\alpha)\right\}$ is attained.

Proof. The idea of the proof is this: by introducing a gap into the spectrum of the kinetic energy term in $H_{l}^{\mathrm{HYL}}$, we can compute a modified pressure which, up to normalization, is the cumulant generating function of $X_{l}(\lambda)$. The expectation of $X_{l}(\lambda)$ is then computed by differentiation and a standard convexity argument used to prove the convergence of the derivative.

Using (6.3), we have

$$
\begin{aligned}
\tilde{\mathbb{E}}_{l}^{\mu}\left[X_{l}(\lambda)\right] & =\left.\left(\beta V_{l}\right)^{-1} \frac{\partial}{\partial s} \tilde{\mathbb{E}}_{l}^{\mu}\left[e^{\beta s V_{l} X_{l}(\lambda)}\right]\right|_{s=0} \\
& =\left.\left(\beta V_{l}\right)^{-1} \frac{\partial}{\partial s} e^{\beta V_{l}\left\{\tilde{p}_{l}(\mu ; s, \lambda)-\tilde{p}_{l}(\mu)\right\}}\right|_{s=0},
\end{aligned}
$$

where $\tilde{p}_{l}(\mu)$ is the pressure corresponding to the hamiltonian $\tilde{H}_{l}$ and $\tilde{p}_{l}(\mu ; s, \lambda)$ is the pressure corresponding to the hamiltonian $\tilde{H}_{l}(s, \lambda)=\tilde{H}_{l}-s V_{l} X_{l}(\lambda)$. Hence we have

$$
\tilde{\mathbb{E}}_{l}^{\mu}\left[X_{l}(\lambda)\right]=\left.\frac{\partial}{\partial s} \tilde{p}_{l}(\mu ; s, \lambda)\right|_{s=0} .
$$


Putting $\widetilde{H}_{l}=H_{l}^{0}+V_{l} u_{l}$, where $H_{l}^{0}$ is the free-gas hamiltonian, we have

$$
\begin{aligned}
e^{\beta V_{l} \tilde{p}_{l}(\mu ; s, \lambda)} & =\sum_{\omega \in \Omega} e^{\beta\left\{\left(\mu N(\omega)+s V_{l} X_{l}(\lambda)-H_{l}^{0}(\omega)-V_{l} u_{l}(\omega)\right\}\right.} \\
& =\sum_{\omega \in \Omega} e^{\beta\left\{(\mu+s) N(\omega)-\hat{H}_{l}(s, \lambda ; \omega)\right\}},
\end{aligned}
$$

where $\widetilde{H}_{l}(s, l)=H_{l}^{0}(s, \lambda)+V_{l} u_{l}$ and $H_{l}(s, \lambda)$ is defined by

$$
H_{l}(s, \lambda ; \omega)=\sum_{\left\{j: \lambda_{l}(j) \leqq j\right\}} \lambda_{l}(i) \sigma_{j}(\omega)+\sum_{\left\{j: \lambda_{l}(j)>\lambda\right\}}\left(s+\lambda_{l}(j) \sigma_{J}(\omega) .\right.
$$

In other words, the hamiltonian $H_{l}^{0}(s, \lambda)$ is the free-gas hamiltonian with $\left\{\lambda_{l}^{\prime}(j)\right\}$ in place of $\left\{\lambda_{l}(j)\right\}$, where

$$
\lambda_{l}^{\prime}(j)=\left\{\begin{array}{lll}
\lambda_{l}(j), & \text { if } \lambda_{l}(j) \leqq \lambda, \\
s+\lambda_{l}(j), & \text { if } \quad \lambda_{l}(j)>\lambda .
\end{array}\right.
$$

It is easy to check that if $0=\lambda_{l}(1) \leqq \lambda_{l}(2) \leqq \ldots$ satisfies (S1) and (S2), then so does $0=\lambda_{l}^{\prime}(1) \leqq \lambda_{l}^{\prime}(2) \leqq \ldots$. Applying these considerations to the HYL-model we have, by Theorem 1, $p^{\mathrm{HYL}}(\mu ; s, \lambda)=\lim _{l \rightarrow \infty} p_{l}^{\mathrm{HYL}}(\mu ; s, \lambda)$ exists. By Theorem 2, we have

$$
p^{\mathrm{HYL}}(\mu ; s, \lambda)= \begin{cases}\inf _{x<0}\left\{\frac{(\mu+s-\alpha)^{2}}{4 a}+p(\alpha ; s, \lambda)\right\}, & \mu \leqq \mu^{*}(s, \lambda), \\ \sup _{\alpha<0}\left\{\frac{(\mu+s-\alpha)^{2}}{4 a}-\frac{\alpha^{2}}{2 a}+p(\alpha ; s, \lambda)\right\}, & \mu \geqq \mu^{*}(s, \lambda),\end{cases}
$$

where $\mu^{*}(s, \lambda)$ is the unique value of $\mu$ for which the two expressions on the righthand side of (6.10) become equal. We note that $s, \lambda \mapsto \mu^{*}(s, \lambda)$ is continuous. Here $p(\alpha ; s, \lambda)$ is the free-gas pressure computed with $H_{l}(s, \lambda ; \omega)$ in place of $H_{l}^{0}(\omega)$; since $p(\alpha)$ can be expressed in terms of the integrated density of states $F(\cdot)$ as

$$
p(\alpha)=\int_{[0, \infty)} p\left(\alpha \mid \lambda^{\prime}\right) d F\left(\lambda^{\prime}\right)
$$

we have

$$
p(\alpha ; s, \lambda)=\int_{[0, \lambda)} p\left(\alpha \mid \lambda^{\prime}\right) d F\left(\lambda^{\prime}\right)+\int_{[\lambda, \infty)} p\left(\alpha \mid s+\lambda^{\prime}\right) d F\left(\lambda^{\prime}\right) .
$$

Since $s \mapsto p_{l}^{\mathrm{HYL}}(\mu ; s, \lambda)$ is convex by a standard argument using Hölder's inequality and $s \mapsto p^{\mathrm{HYL}}(\mu ; s, \lambda)$ is differentiable at $s=0$ provided $\mu \neq \mu^{*}(0, \lambda)$ we have, by Griffith's inequality in the form proved by Hepp and Lieb [14],

$$
\left.\lim _{l \rightarrow \infty} \frac{\partial p_{l}^{\mathrm{HYL}}}{\partial s}(\mu ; s, \lambda)\right|_{s=0}=\left.\frac{\partial}{\partial s} p^{\mathrm{HYL}}(\mu ; s, \lambda)\right|_{s=0} .
$$

Using (6.6), (6.13), and (6.10), we have finally

$$
\Delta(\mu)= \begin{cases}-\alpha(\mu) / a, & \mu>\mu^{*}, \\ 0, & \mu<\mu^{*},\end{cases}
$$

where $\alpha(\mu)$ is the unique value of $\alpha$ at which the supremum in (5.1) is attained. 


\section{Appendix. Large Deviation Results for the Free-Boson Gas}

In this section, we provide the proofs of the large deviation results used in Sects. 3 and 4.

Theorem A1. Suppose that (S1) and (S2) hold; then, for $\mu<0$, the sequence

$$
\left\{\mathbb{K}_{l}^{\mu}=\mathbb{P}_{l}^{\mu} \circ X_{l}^{-1}: l=1,2, \ldots\right\}
$$

of distributions of the particle number density $X_{l}=N / V_{l}$ in the free-boson gas satisfies the large deviation principle with constants $\left\{V_{l}\right\}$ and rate-function

$$
I^{\mu}(x)=p(\mu)+f(x)-\mu x .
$$

Proof. It follows from (3.6) that the following formula holds provided $\mu$ and $\mu+t$ both lie in $(-\infty, 0)$ :

$$
\int_{[0 . \infty)} e^{\beta V_{l} t x} \mathbb{K}_{l}^{\mu}[d x]=e^{\beta V_{l}\left\{p_{l}(\mu+t)-p_{l}(\mu)\right\}} ;
$$

by Proposition 1, we have

$$
C^{\mu}(t) \stackrel{\text { def }}{=} \lim _{l \rightarrow \infty} \frac{1}{\beta V_{l}} \int_{[0, \infty)} e^{\beta V_{l} t x} \mathbb{K}_{l}^{\mu}[d x]=p(\mu+t)-p(\mu) .
$$

The verification of (LD1), (LD2), and (LD3) is now routine (see [5] for a review of the general principles); to make the paper self-contained, we sketch the proof. The rate-function (A1) is the Legendre transform of $C^{\mu}(t)$, given by (A3):

$$
I^{\mu}(x)=\sup _{\{t: t+\mu<0\}}\left\{t x-C^{\mu}(t)\right\} .
$$

The properties (LD1) and (LD2) follow from (A4). To verify (LD3), consider first the case in which the closed set $C$ is an interval $J_{1}=\left[0, \varrho_{1}\right]$ with $\varrho_{1}<p^{\prime}(\mu)$; for each $l$ and each $x<0$, we have

$$
\begin{aligned}
\mathbb{K}_{l}^{\mu}\left[J_{l}\right] & =\int_{[0, \infty)} 1_{\left[0, \varrho_{1} l\right.}(x) \mathbb{K}_{l}^{\mu}[d x] \leqq \int_{[0, \infty)} e^{\alpha \beta V_{l}\left(x-\varrho_{1}\right)} \mathbb{K}_{l}^{\mu}[d x] \\
& =e^{\beta V_{l}\left\{p_{l}(\mu+\alpha)-p_{l}(\mu)-\alpha \varrho_{1}\right\}} .
\end{aligned}
$$

It then follows from Proposition 1 that

$$
\lim _{l \rightarrow \infty} \sup \frac{1}{\beta V_{l}} \ln \mathbb{K}_{l}^{\mu}\left[J_{1}\right] \leqq \inf _{\alpha<0}\left\{p(\mu+\alpha)-p(\mu)-\alpha \varrho_{1}\right\}=-I^{\mu}\left(\varrho_{1}\right), \quad \text { by (A4) . }
$$

It follows in analogous fashion, in the case in which $C$ is an interval $\left[\varrho_{2}, \infty\right)$ with $\varrho_{2}>p^{\prime}(\mu)$, that

$$
\limsup _{l \rightarrow \infty} \frac{1}{\beta V_{l}} \ln \mathbb{K}_{l}^{\mu}\left[J_{2}\right] \leqq-I^{\mu}\left(\varrho_{2}\right) .
$$

A simple argument (see p. 8 of [5]) then shows that for an arbitrary closed subset $C$ of $[0, \infty)$

$$
\limsup _{l \rightarrow \infty} \frac{1}{\beta V_{l}} \ln \mathbb{K}_{l}^{\mu}[C] \leqq-\inf _{C} I^{\mu}(x)
$$


It remains to prove that (LD4) holds. First note that the measures $\mathbb{K}_{l}^{\mu}, \mathbb{K}_{l}^{\mu+t}$, with $\mu<0$ and $\mu+t<0$, are mutually absolutely continuous:

$$
\mathbb{K}_{l}^{\mu+t}[d x]=e^{\beta V_{l} C_{l}^{\mu}(x ; t)} \mathbb{K}_{l}^{\mu}[d x],
$$

where

$$
C_{l}^{\mu}(x ; t)=t x+p_{l}(\mu)-p_{l}(\mu+t) .
$$

[To check this, compute the Laplace transforms of both sides of (A5).] Now let $G$ be an arbitrary open subset of $[0, \infty)$; let $y$ be an arbitrary point of $G$ and choose $\delta>0$ so that $B_{y}^{\delta}=(y-\delta, y+\delta)$ is contained in $G$. Then

$$
\mathbb{K}_{l}^{\mu}[G] \geqq K_{l}^{\mu}\left[B_{y}^{\delta}\right] .
$$

Now, for $l=1,2, \ldots$, choose $t_{l}$ so that $p_{l}^{\prime}\left(\mu+t_{l}\right)=y$ using (A5) we have

$$
\begin{aligned}
\mathbb{K}_{l}^{\mu}\left[B_{y}^{\delta}\right] & =\int_{B_{y}^{\delta}} \mathbb{K}_{l}^{\mu}[d x]=\int_{B_{y}^{\delta}} e^{-\beta V_{l} C_{l}^{\mu}\left(x ; t_{l}\right)} \mathbb{K}_{l}^{\mu+t_{l}}[d x] \\
& \geqq e^{-\beta V_{l}\left\{C_{l}^{\mu}\left(v ; t_{l}\right)+\delta\left|t_{l}\right|\right\}} \mathbb{K}_{l}^{\mu+t}\left[B_{y}\right] .
\end{aligned}
$$

By Proposition 2, $\mu+t_{l} \rightarrow \mu(y)$ and $p_{l}\left(\mu+t_{l}\right) \rightarrow p(\mu(y))$ as $l \rightarrow \infty$; hence $C_{l}^{\mu}\left(y ; t_{l}\right)$ $\rightarrow C^{\mu}(y ; \mu(y)-y)$. Thus

$$
\begin{aligned}
\liminf _{l \rightarrow \infty} \frac{1}{\beta V_{l}} \ln \mathbb{K}_{l}^{\mu}[G] \geqq & -C^{\mu}(y ; \mu(y)-y)-\delta|\mu(y)-\mu| \\
& +\liminf _{l \rightarrow \infty} \frac{1}{\beta V_{l}} \ln \mathbb{K}_{l}^{\mu+t_{l}}\left[B_{y}^{\delta}\right] .
\end{aligned}
$$

We will show that

$$
\liminf _{l \rightarrow \infty} \frac{1}{\beta V_{l}} \ln \mathbb{K}_{l}^{\mu+t_{l}}\left[B_{y}^{\delta}\right]=0
$$

it will then follow that (LD4) holds.

For arbitrary open $G$, we have

$$
\liminf _{l \rightarrow \infty} \frac{1}{\beta V_{l}} \ln \mathbb{K}_{l}^{\mu}[G] \geqq-\inf _{G} I^{\mu}(y),
$$

because $\delta>0$ was arbitrarily small and $y$ was an arbitrary point of $G$. To prove (A8) we distinguish two cases: if $y<\varrho_{c}$ then the convexity of $\mu \mapsto p_{l}(\mu)$ together with Griffith's lemma in the version proved by Hepp and Lieb [14] allows us to conclude that

$$
\lim _{l \rightarrow \infty} \int_{[0, \infty)} e^{s x} \mathbb{K}_{l}^{\mu+t_{l}}[d x]=e^{s p^{\prime}(\mu(y))}=e^{s v},
$$

so that $\left\{\mathbb{K}_{l}^{\mu+t_{l}}\right\}$ converges weakly to the degenerate distribution concentrated at $y$ and hence, for $l$ sufficiently large, $\mathbb{K}_{l}^{\mu+t_{l}}\left[B_{y}^{\delta}\right]>\frac{1}{2}$ and (A8) follows; if $y \geqq \varrho_{c}$ then $\mu(y)=0$ and we must proceed differently.

Lemma A1. Let $N_{1}$ and $N_{2}$ be independent non-negative integer valued random variables with means $m_{1}$ and $m_{2}$, respectively. Suppose that $N_{1}$ is geometrically 
distributed and that $\delta \geqq 1$; then

$$
\mathbb{P}\left[N_{1}+N_{2} \in B_{m_{1}+m_{2}}^{\delta}\right] \geqq \frac{1}{m_{1}+m_{2}}\left(\frac{m_{1}}{m_{1}+1}\right)^{m_{1}+m_{2}+2} .
$$

Proof. First note that the interval $B_{m_{1}+m_{2}}^{1}=\left(m_{1}+m_{2}-1, m_{1}+m_{2}+1\right)$ contains a unique integer greater than $m_{1}+m_{2}$ which we denote by $n_{0}$. Now

$$
\begin{aligned}
\mathbb{P}\left[N_{1}+N_{2} \in B_{m_{1}+m_{2}}^{\delta}\right] & =\sum_{m \in B_{m_{1}-m_{2}}^{\delta_{1}}} \sum_{n=0}^{m} \mathbb{P}\left[N_{1}=m-n\right] \mathbb{P}\left[N_{2}=n\right] \\
& \geqq \sum_{n=0}^{n_{0}} \mathbb{P}\left[N_{1}=n_{0}-n\right] \mathbb{P}\left[N_{2}=n\right] .
\end{aligned}
$$

Since, by assumption, $N_{1}$ is geometrically distributed we have

$$
\mathbb{P}\left[N_{1}=n\right]=\left(m_{1}+1\right)^{-1}\left(\frac{m_{1}}{m_{1}+1}\right)^{n} .
$$

Clearly, $n \mapsto \mathbb{P}\left[N_{1}=n\right]$ is a decreasing function so that

$$
\sum_{n=0}^{n_{0}} \mathbb{P}\left[N_{1}=n_{0}-n\right] \mathbb{P}\left[N_{2}=n\right] \geqq \mathbb{P}\left[N_{1}=n_{0}\right] \mathbb{P}\left[N_{2} \leqq n\right] ;
$$

substituting from (A13), we have

$$
\sum_{n=0}^{n_{0}} \mathbb{P}\left[N_{1}=n_{0}-n\right] \mathbb{P}\left[N_{2}=n\right] \geqq \frac{1}{m_{1}+1}\left(\frac{m_{1}}{m_{1}+1}\right)^{m_{1}+m_{2}+1} \mathbb{P}\left[N_{2} \leqq n_{0}\right] \text {. }
$$

But, by Markov's inequality, we have

$$
\mathbb{P}\left[N_{2} \leqq n_{0}\right] \geqq \mathbb{P}\left[N_{2} \leqq m_{1}+m_{2}\right] \geqq \frac{m_{1}}{m_{1}+m_{2}}
$$

Hence

$$
\mathbb{P}\left[N_{1}+N_{2} \in B_{m_{1}+m_{2}}^{\delta}\right] \geqq \frac{1}{m_{1}+m_{2}}\left(\frac{m_{1}}{m_{1}+1}\right)^{m_{1}+m_{2}+2}
$$

Returning to the proof of Theorem A1, we see that for $\sup _{j \geqq 1} s_{j}<-\mu$ we have

$$
\mathbb{E}_{l}^{\mu}\left[e^{\beta\left(s_{1} \sigma_{1}+s_{2} \sigma_{2}+\ldots\right)}\right]=\prod_{j \geqq 1} \frac{1-e^{\beta\left(\mu-\lambda_{l}(j)\right)}}{1-e^{\beta\left(\mu-\lambda_{l}(j)+s_{J}\right)}},
$$

from which we conclude that $\left\{\sigma_{j}: j=1,2, \ldots\right\}$ is a sequence of independent geometrically distributed random variables. Applying Lemma A1 with $N_{1}=\sigma_{1}$ and $N_{2}=N-\sigma_{1}$, so that $\frac{m_{1}}{m_{1}+1}=e^{\beta\left(\mu+t_{l}\right)}$ and $m_{1}+m_{2}=V_{l} y$, we have

$$
\mathbb{K}_{l}^{\mu+t_{l}}\left[B_{y}^{\delta}\right] \geqq \frac{1}{V_{l} y}-e^{\beta\left(\mu+t_{l}\right)\left(V_{l} y+2\right)}
$$

for $V_{l} \geqq 1 / \delta$. It follows that 


$$
\liminf _{l \rightarrow \infty} \frac{1}{\beta V_{l}} \ln \mathbb{K}_{l}^{\mu+t_{l}}\left[B_{y}^{\delta}\right]=0
$$

since, for $y \geqq \varrho_{c}, \mu+t_{l} \rightarrow 0$. Thus we have, in both cases,

$$
\liminf _{l \rightarrow \infty} \frac{1}{\beta V_{l}} \ln \mathbb{K}_{l}^{\mu}[G] \geqq-p(\mu)-f(y)+\mu y=-I^{\mu}(y)
$$

for all $y$ in $G$, since $\delta$ was arbitrary. Hence

$$
\liminf _{l \rightarrow \infty} \frac{1}{\beta V_{l}} \ln \mathbb{K}_{l}^{\mu}[G] \geqq \sup _{G}\left(-I^{\mu}(y)\right)=-\inf _{G} I^{\mu}(y) .
$$

The large deviation result established above enables us to apply Varadhan's theorem to suitable functions of $X_{l}=N / V_{l}$; to deal with functions of the two variables $\sigma_{1} / V_{l}$ and $N / V_{l}$, we prove a large deviation result for the sequence of probability distribution of a vector-valued random variable. Define the vectorvalued random variable $X_{l}: \Omega \rightarrow \mathbb{R}^{2}$ by $X_{l}^{(1)}=V_{l}^{-1} \sigma_{1}, X_{l}^{(2)}=V_{l}^{-1} \sum_{j \geqq 2} \sigma_{\jmath}$, with cumulant generating function $C_{l}^{\mu}[\cdot]$ by

$$
C_{l}^{\mu}[t]=\left(\beta V_{l}\right)^{-1} \ln \mathbb{E}_{l}^{\mu}\left[e^{\beta V_{l}\left\langle t \cdot X_{l}\right\rangle}\right]
$$

In order to prove a large deviation result for $\mathbb{K}_{l}^{\mu}=\mathbb{P}_{l}^{\mu} \circ X_{l}^{-1}$, it is necessary to make a further hypothesis about the single-particle spectrum.

Lemma A2. Suppose that (S1) and (S2) hold and that $\lim _{l \rightarrow \infty} \hat{\lambda}_{l}(2)=\lambda(2)$ exists and is a point of continuity of $F$; then the cumulant generating function $C^{\mu}[t]=\lim _{l \rightarrow \infty} C_{l}^{\mu}[t]$ exists for $\mu<0$ and all $t$ in $\mathbb{R}_{+}^{2}$ and is given by

$$
C^{\mu}[t]= \begin{cases}p\left(\mu+t_{2}\right)-p(\mu), & t \in \mathscr{D}^{\mu} \\ \infty, & \text { otherwise }\end{cases}
$$

where

$$
\mathscr{D}^{\mu}=\left\{t \in \mathbb{R}_{+}^{2}: \mu+t_{2}-\lambda(2)<0, \mu+t_{1}<0\right\} .
$$

Proof. We have

$$
C_{l}^{\mu}[t]=-p_{l}(\mu)+p_{l}^{(1)}\left(\mu+t_{1}\right)+p_{l}^{(2)}\left(\mu+t_{2}\right)
$$

where

$$
p_{l}^{(1)}(\alpha)=-\left(\beta V_{l}\right)^{-1} \ln \left(1-e^{-\beta \alpha}\right), \quad \alpha<0,
$$

and

$$
p_{l}^{(2)}(\alpha)=-\left(\beta V_{l}\right)^{-1} \sum_{j \geqq 2} \ln \left(1-e^{-\beta\left(\alpha-\lambda_{l}(j)\right)}\right), \quad \alpha<\lambda_{l}(2) ;
$$

clearly $\lim _{l \rightarrow \infty} p_{l}^{(1)}(\alpha)=0$.

Define $\tilde{\lambda}_{l}(j)=\lambda_{l}(j+1)-\lambda_{l}(2), j=1,2, \ldots$; then $\left\{\tilde{\lambda}_{l}(j): j=1,2, \ldots\right\}$ satisfies (S1) and (S2) and the corresponding density of states $\widetilde{F}$ is given by $\widetilde{F}(\lambda)=F(\lambda+\lambda(2))$. 
Since

$$
p_{l}^{(2)}(\alpha)=\int_{[0, \infty)} p\left(\alpha-\lambda_{l}(2) \mid \lambda_{)}\right) d \tilde{F}_{l}(\lambda)
$$

we have, by Proposition 3 of Sect. 1,

$$
\lim _{l \rightarrow \infty} p_{l}^{(2)}(\alpha)=\int_{[0, \infty)} p(\alpha-\lambda(2) \mid \lambda) d \widetilde{F}(\lambda)=\int_{[\lambda(2), \infty)} p(\alpha \mid \lambda) d F(\lambda)=p(\alpha)
$$

for $\alpha<\lambda(2)$. It follows that

$$
\lim _{l \rightarrow \infty} C_{l}^{\mu}[t]=p\left(\mu+t_{2}\right)-p(\mu)
$$

for $t$ in $\mathscr{D}^{\mu}$; put $C^{\mu}[t]=\infty$ for $t$ in the complement of $\mathscr{D}^{\mu}$; then $t \mapsto C^{\mu}[t]$ is a closed proper convex function on $\mathbb{R}^{2}$ with $\operatorname{dom} C^{\mu}=\mathscr{D}^{\mu}$. Put

$$
I^{\mu}[x]=\sup _{t \in \mathbb{R}^{2}}\left\{\langle x, t\rangle-C^{\mu}[t]\right\}
$$

then we have

Theorem A2. Suppose that (S1) and (S2) hold and that $\lambda(2)=\lim \lambda_{l}(2)$ exists and is a point of continuity of $F$. Then, for each $\mu<0$, the sequence $\left\{\mathbb{K}_{l}^{\mu}: l=1,2, \ldots\right\}$ satisfies the large deviation principle with constants $\left\{V_{l}\right\}$ and rate-function $I^{\mu}(\cdot)$ given by

$$
I^{\mu}(x)=p(\mu)+f\left(x_{2} ; \lambda(2)\right)-\mu\left(x_{1}+x_{2}\right), \quad x_{1} \geqq 0, \quad x_{2} \geqq 0 .
$$

Proof. The proof that (LD1) and (LD2) hold follows, as in the proof of Theorem A1, from the fact that $I^{\mu}(\cdot)$ is the Legendre transform of $C^{\mu}(\cdot)$. To prove that (LD3) holds, we follows Ellis [13] and adapt to our situation

Gärtner's Lemma. Let $K$ be a non-empty closed subset of $\mathbb{R}^{2}$ define $I^{\mu}[K]$ $=\inf _{k} I^{\mu}(x)$. If $0<I^{\mu}[K]<\infty$ then there exists a finite set $\tau^{(1)}, \ldots, \tau^{(r)}$ of non-zero vectors in $\mathbb{R}^{2}$ such that, for $\varepsilon>0$ and $c=I^{\mu}[K]-\varepsilon$,

$$
K \subset \bigcup_{j=1}^{r} H_{+}^{\mu}\left(\tau^{(j)} ; c\right)
$$

where $H_{+}^{\mu}(\tau ; c)=\left\{x:\langle x, \tau\rangle-C^{\mu}(\tau) \geqq c\right\}$; if $I^{\mu}[K]=+\infty$ then, for each $R>0$, there exists a finite set $\tau^{(1)}, \ldots, \tau^{(r)}$ of non-zero vectors in $\mathbb{R}^{2}$ such that

$$
K \subset \bigcup_{j=1}^{R} H_{+}^{\mu}\left(\tau^{(j)} ; R\right) .
$$

First suppose that $K$ is such that $0<I^{\mu}[K]<\infty$; then

$$
\mathbb{K}_{l}^{\mu}[K] \leqq \sum_{j=1}^{r} \mathbb{K}_{l}^{\mu}\left[H_{+}^{\mu}\left(\tau^{(j)} ; c\right)\right]
$$

By Markov's inequality,

$$
\begin{aligned}
\mathbb{K}_{l}^{\mu}\left[H_{+}^{\mu}\left(\tau^{(j)} ; c\right)\right] & \leqq e^{-e \beta V_{l}\left\{C^{\mu}\left(\tau^{(j)}\right)+c\right\}} \int_{\substack{\mathbb{R}_{+}^{2} \\
\beta V_{l}\left\langle x, \tau^{(j)}\right\rangle}} \mathbb{K}_{l}^{\mu}[d x] \\
& \leqq e^{-\beta V_{l}\left\{C^{\mu}\left(\tau^{(j)}\right)+c-C_{l}^{\mu}\left(\tau^{(j)}\right)\right\}}
\end{aligned}
$$


hence

$$
\limsup _{l \rightarrow \infty} \frac{1}{\beta V_{l}} \ln \mathbb{K}_{l}^{\mu}\left[H_{+}^{\mu}\left(\tau^{(j)} ; c\right)\right]=-I^{\mu}[K],
$$

since $\varepsilon>0$ was arbitrary and $C_{l}^{\mu}(t) \rightarrow C^{\mu}(t)$ by Lemma A2. It then follows from (A26) that (LD3) holds in the case $0<I^{\mu}[K]<\infty$; if $I^{\mu}[K]=+\infty$ then $\lim \sup \frac{1}{\beta V_{l}} \ln \mathbb{K}_{l}^{\mu}[K] \leqq-R$ for each $R>0$ and again (LD3) holds.

It remains to verify (LD4); let $G$ be an arbitrary point in $G$ and choose $\delta>0$ such that $\left(y_{1}-\delta, y_{1}+\delta\right) \times\left(y_{2}-\delta, y_{2}+\delta\right) \subset G$. Then

$$
\mathbb{K}_{l}^{\mu}[G] \geqq \prod_{j=1}^{2} \mathbb{K}_{l}^{(j) \cdot \mu}\left[\left(y_{j}-\delta, y_{j}+\delta\right)\right],
$$

where $\mathbb{K}_{l}^{(j), \mu}$ is determined by

$$
\int_{[0, \infty)} e^{\beta V_{l} t_{j} x} \mathbb{K}_{l}^{(j), \mu}[d x]=e^{\beta \boldsymbol{V}_{l}\left\{p_{l}^{(\prime)}\left(\mu+t_{j}\right)-p_{l}^{(J)}(\mu)\right\}} .
$$

Now

$$
\liminf _{l \rightarrow \infty}\left(\beta V_{l}\right)^{-1} \ln \mathbb{K}_{l}^{(2), \mu}\left[\left(y_{2}-\delta, y_{2}+\delta\right] \geqq-I^{(2), \mu}\left(y_{2}\right),\right.
$$

where

$$
I^{(2) \cdot \mu}(x)=\sup _{\mu+t_{2}<\lambda(2)}\left\{p(\mu)-p\left(\mu+t_{2}\right)+t_{2} x\right\}=p(\mu)+f(x ; \lambda(2))-\mu x, \quad x \geqq 0,
$$

by the reasoning used in the proof of Theorem A1. Finally,

$$
\lim \inf \left(\beta V_{l}\right)^{-1} \ln \mathbb{K}_{l}^{(1), \mu}\left[\left(y_{1}-\delta, y_{1}+\delta\right)\right] \geqq-I^{(1) \cdot \mu}\left(y_{1}\right),
$$

by direct calculation, where $I^{(1) \cdot \mu}(x)=-\mu x, x \geqq 0$ and (LD4) holds since $y$ was chosen arbitrarily in $G$.

Acknowledgement. The authors acknowledge their indebtedness to Dr. T. C. Dorlas for his constructive criticism of an earlier version of this paper.

\section{References}

1. London, F.: On the Bose-Einstein condensation. Phys. Rev. 54, 947-954 (1938)

2. London, F.: Superfluids, Vol. II. New York: Wiley 1954

3. Huang, K., Yang, C.N., Luttinger, J.M.: Imperfect bose gas with hard-sphere interactions. Phys. Rev. 105, 776-784 (1957)

4. Thouless, D.J.: The quantum-mechanics of many-body systems. New York: Academic Press 1961

5. Lewis, J.T.: Why do bosons condense? In: Statistical mechanics and field theory: mathematical aspects proceedings, Groningen 1985. Dorlas, T.C., Hugenholtz, N.M., Winnink, M. (eds.). Lecture Notes in Physics, Vol. 257. Berlin, Heidelberg, New York: Springer 1986

6. Varadhan, S.R.S.: Asymptotic probabilities and differential equations. Commun. Pure Appl. Math. 19, 261-286 (1966)

7. Berg, M. van den, Lewis, J.T., Pulé, J.V.: A general theory of Bose-Einstein condensation. Helv. Phys. Acta 59, 1271-1288 (1986) 
8. Berg, M. van den: On boson condensation into an infinite number of low-lying levels. J. Math. Phys. 23, 1159-1161 (1982)

9. Berg, M. van den, Lewis, J.T.: On the free Boson gas in a weak external potential. Commun. Math. Phys. 81, 475-494 (1981)

10. Berg, M. van den, Lewis, J.T., Smedt, P. de: Condensation in the imperfect Boson gas. J. Stat. Phys. 37, 697-707 (1984)

11. Hove, L. van: Math. Revs. 18, 836 (1957)

12. Critchley, R.H.: Approximate equilibrium states for two models of an interacting Boson gas. J. Math. Phys. 21, 359-363 (1980)

13. Ellis, R.: Entropy, large deviations and statistical mechanics. Berlin, Heidelberg, New York: Springer 1985

14. Hepp, K., Lieb, E.H.: Equilibrium statistical mechanics of matter interacting with the quantized radiation field. Phys. Rev. A 8, 2517-2525 (1973)

Communicated by J. Fröhlich

Received August 18, 1986; in revised form February 22, 1988 
